\title{
The Rare but Fatal Connection: Atrio-Esophageal Fistula
}

\author{
Tom Kai Ming Wang and Jen-Li Looi* \\ Department of Cardiology, Middlemore Hospital, Auckland, New Zealand
}

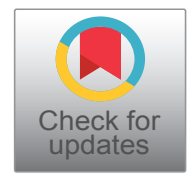

*Corresponding author: Jen-Li Looi, Department of Cardiology, Middlemore Hospital, Private Bag 933111, Otahuhu, Auckland, New Zealand, Tel: +649-2760061, Fax: +649-2709746

A 64-year-old man underwent catheter ablation for atrial fibrillation (AF) a month ago, which involved isolation of pulmonary veins with wide area circumferential ablation and posterior left atrial wall with roof and inferior line at 30-35 watts radiofrequency energy delivery. He presented initially with fever and palpitation, but subsequently collapsed with ventricular tachycardia and transient lateral ST elevation on electrocardiogram, and developed dysphasia with right arm weakness. Transthoracic echocardiogram showed severe biventricular systolic impairment. Coronary angiography was unremarkable. CT chest showed outpouching of the posterior wall of the left atrium (Figure 1A) with gas locules (Figure 1B) adjacent to the esophagus, in keeping with an atrio-esophageal fistula. His electrocardiogram findings and neurological symptoms are likely due to air embolism.

He was admitted to intensive care and intravenous tazocin and vancomycin were initiated. Gastroscopy confirmed a $10 \mathrm{~mm}$ fistula in the middle third of the esophagus (Figure 1C). A $30 \times 135 \mathrm{~mm}$ covered stent was placed as a temporary measure. Unfortunately, he developed progressive multi-organ failure, and cardiac surgery was felt to be at prohibitive risk. He passed away a week after presentation.

Atrio-esophageal fistula (AEF) is a rare but often fatal complication of percutaneous AF ablation [1]. Use of general anesthesia, ablation time, temperature and power settings, and ablation over the posterior wall are hypothesized as potential contributors to the development of AEF [2]. Symptoms include chest pain, sepsis, gastrointestinal bleeding and neurological symptoms, with the latter two being reported as multivariable predictors of mortality [3].
Contrast esophagram or CT chest are useful for diagnosis. A systematic review of 87 patients found high mortality rates of $96 \%$ with medical management, $100 \%$ with esophageal stent and $33 \%$ with surgical closure [3]. The time from procedure to clinical presentation of AEF is highly varied, thus clinical suspicion of AEF should persist for at least the first 2 months post-AF ablation in the context of relevant signs and symptoms. Awareness of the variable clinical presentations of AEF post-AF ablation is paramount for all clinicians. This case illustrates the importance of recognizing the cluster of symptoms post-AF ablation to enable early diagnosis and surgical intervention [4].

\section{References}

1. Cappato R, Calkins H, Chen SA, Davies W, lesaka $Y$, et al. (2010) Updated worldwide survey on the methods, efficacy, and safety of catheter ablation for human atrial fibrillation. Circ Arrhythm Electrophysiol 3: 32-38.

2. Nair GM, Nery PB, Redpath CJ, Lam BK, Birnie DH (2014) Atrioesophageal fistula in the era of atrial fibrillation ablation: a review. Can J Cardiol 30: 388-395.

3. Han HC, Ha FJ, Sanders P, Spencer R, Teh AW, et al. (2017) Atrioesophageal Fistula: Clinical Presentation, Procedural Characteristics, Diagnostic Investigations, and Treatment Outcomes. Circ Arrhythm Electrophysiol 10: $1-12$.

4. Yousuf T, Keshmiri H, Bulwa Z, Kramer J, Sharjeel Arshad $\mathrm{HM}$, et al. (2016) Management of Atrio-Esophageal Fistula Following Left Atrial Ablation. Cardiol Res 7: 36-45. in any medium, provided the original author and source are credited. 


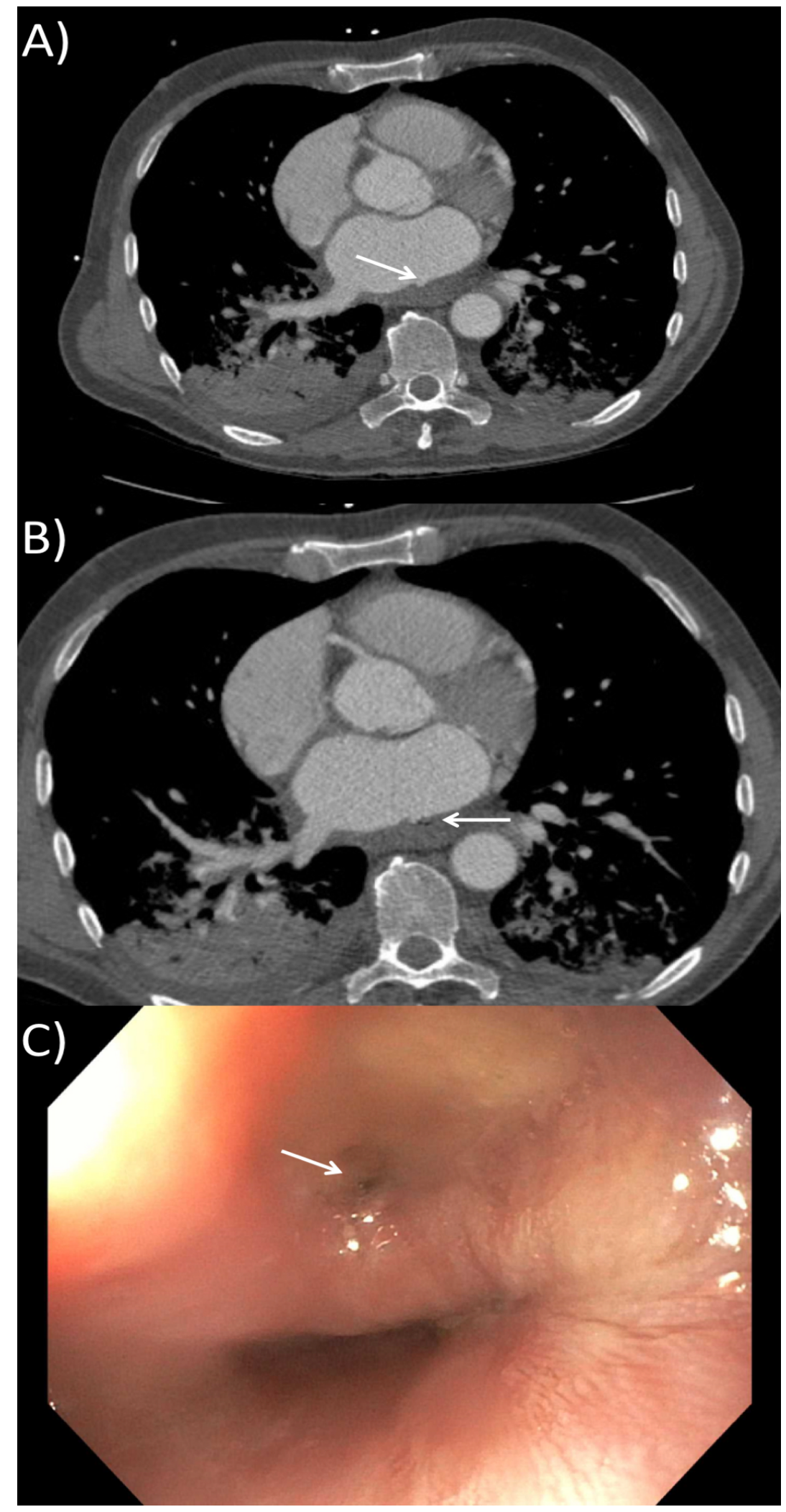

Figure 1: A) CT showing left atrial posterior outpouching (white arrow) on axial view, the thickened posterior wall of the left atrium suggests; B) CT showing left atrial posterior outpouching (white arrow) and adjacent gas locules (black arrow) next to the esophagus on axial view; C) Atrio-esophageal fistula on gastroscopy.

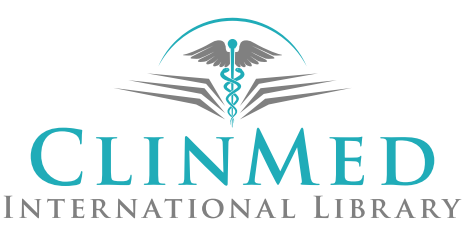

